\title{
Extension of extramammary Paget disease of the vulva to the cervix
}

\author{
J Lloyd, D J Evans, A M Flanagan
}

\begin{abstract}
Extramammary Paget disease of the vulva was found in association with vulval adenocarcinoma in an elderly woman who also had a uterine prolapse. The characteristic histological appearances of extramammary Paget disease were masked by striking reactive changes in the squamous epithelium. Primary excision of both the intraepithelial and invasive disease appeared complete. However, a subsequent hysterectomy with repair of the prolapse revealed extramammary Paget disease in the upper vaginal mucosa and cervix, a finding which is very rarely described. Pathogenesis and diagnosis of extramammary Paget disease is discussed, with differential diagnosis and reference to immunohistochemical methods.

(F Clin Pathol 1999;52:538-540)
\end{abstract}

Histopathology, St

Mary's Hospital NHS

Trust, Praed Street,

London W2 1NY, UK

J Lloyd

Imperial College School of Medicine at St Mary's, Norfolk

Place, London

W2 1PG, UK

D J Evans

A M Flanagan

Correspondence to:

Dr Lloyd.

email: a.flanagan@ic.ac.uk

Keywords: vulva; Paget disease; extramammary Paget disease; cervix

\section{Case report}

An 88 year old woman was referred to the gynaecology department with the diagnosis of a vulval tumour and uterine prolapse with profuse vulval discharge. The patient had received surgical treatment eight years earlier for invasive adenocarcinoma of the vulva, but the original histology was not available for assessment. Clinical examination revealed a large tumour arising on the left side of the vulva with associated thickening and scaling of the surrounding skin. Symptomatic prolapse of the uterus was also noted. Incisional biopsy of the

Accepted for publication 16 March 1999

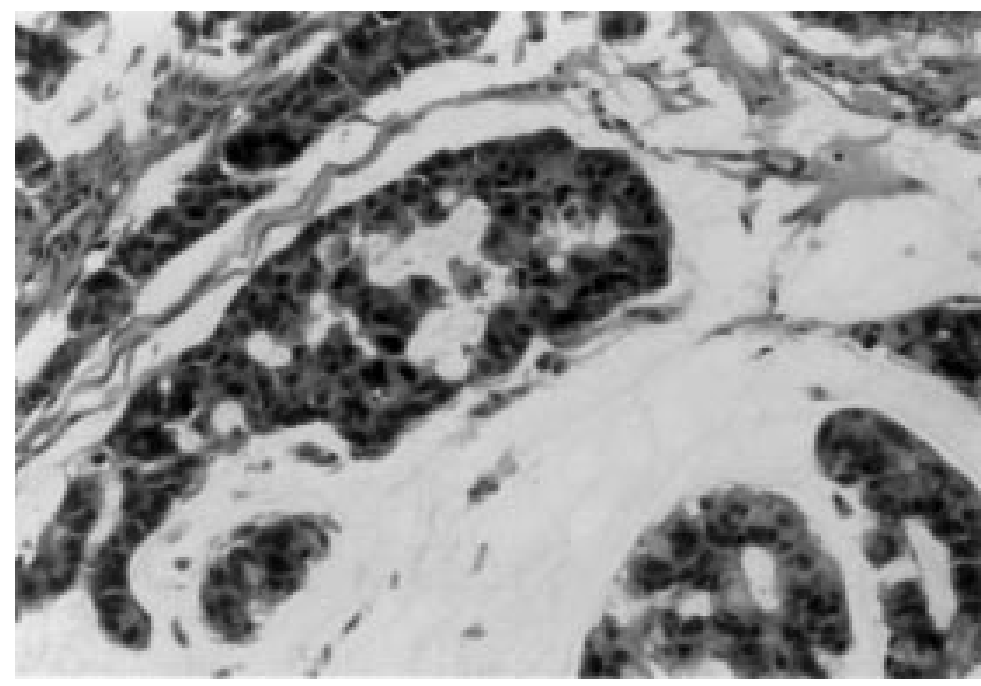

Figure 1 Section of vulval adenocarcinoma with glands embedded in pools of mucin. (Haematoxylin and eosin, $\times 248$.) tumour revealed the presence of invasive, poorly differentiated adenocarcinoma and the patient was admitted for radical vulvectomy, bilateral groin lymphadenectomy, and split skin grafting.

\section{Pathological findings}

The vulvectomy specimen was a crescent shaped piece of non-pigmented skin $(17 \mathrm{~cm}$ lateral dimension, $8 \mathrm{~cm}$ anteroposterior) with a greater proportion of the skin lying to the left of the introitus. A firm, indurated, raised white tumour $(2 \times 1 \times 1 \mathrm{~cm})$ was present $2 \mathrm{~cm}$ to the left of the introitus. The tumour was at least 3 $\mathrm{cm}$ clear of the deep resection margin. Much of the skin around the tumour was rough and fissured and displayed multiple papillary projections. The skin at the introitus was glazed and shiny, suggesting atrophy. A $0.5 \mathrm{~cm}$ rim of macroscopically normal skin was present around the lateral resection margin.

The histological diagnosis of an adenocarcinoma made on the original biopsy was confirmed. Some areas showed mucin production (fig 1), others had prominent signet ring cells, while other areas were less well differentiated. The abnormal skin adjacent to and overlying the tumour showed marked squamous hyperplasia with dramatic elongation of the rete ridges and striking keratinisation (fig 2). The epidermis had an atypical appearance and close inspection showed that it was infiltrated by a population of pale granular cells with large, pleomorphic nuclei and prominent nucleoli. The cells were concentrated in the basal epidermis but staining for mucin and epithelial membrane antigen (EMA) revealed that numbers of them were also were present in the upper strata. A diagnosis of extramammary Paget disease was made. The Paget disease was multifocal, with isolated foci in the surrounding vulval skin and within the vaginal mucosa, which appeared not to be in continuity with the main lesion. The invasive carcinoma was completely excised at all margins and the intraepidermal component appeared to be clear of the vaginal resection margin by at least $1 \mathrm{~cm}$ in multiple sections examined.

Four weeks after the vulvectomy the skin grafts were sufficiently healed to permit vaginal hysterectomy and repair of the prolapse. The uterus and cervix ( $7 \mathrm{~cm}$ fundus- 0 , $3 \mathrm{~cm}$ tubetube, $2 \mathrm{~cm}$ anteroposterior) with attached cuff of vaginal tissue was received in the department of histopathology. The vaginal mucosa, endometrium, and myometrium appeared normal. The cervix was unremarkable except for a small area of ulceration $(0.5 \mathrm{~cm}$ diameter) on the left side. The cervix and vaginal tissue were 


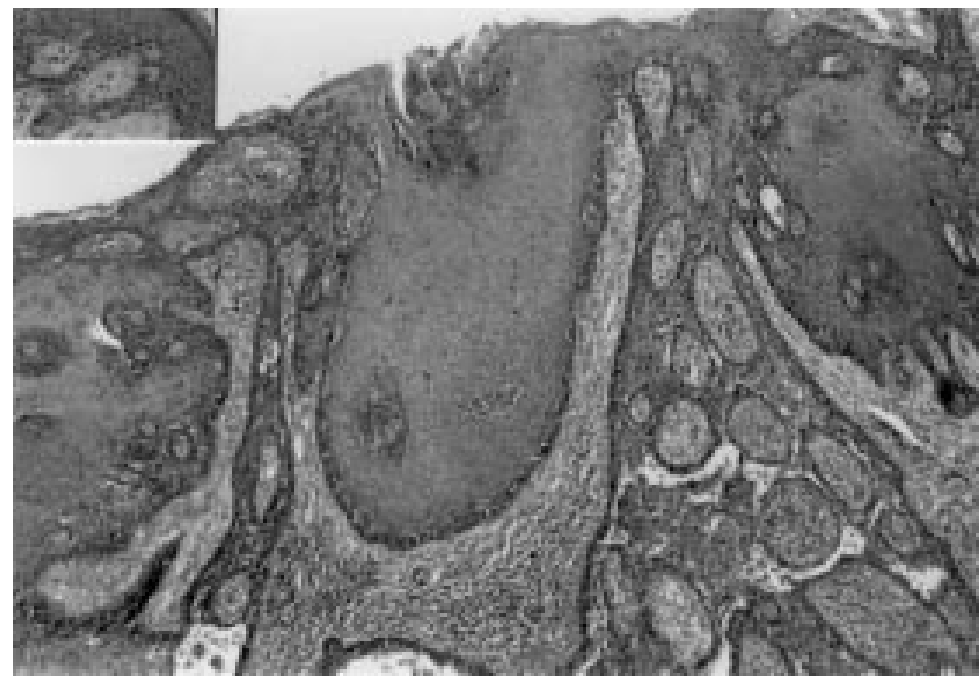

Figure 2 Low power photomicrograph of vulval skin with hyperkeratosis and striking epithelial hyperplasia. High power view (inset) shows infiltration of the epithelium by atypical granular cells consistent with extramammary Paget disease.

sampled, revealing extramammary Paget disease in all quadrants of the vaginal mucosa, focally extending to the cervical transformation zone (fig 3). The area of cervical ulceration also showed infiltration by atypical cells and Paget disease was present at much of the vaginal resection margin. The endometrium was atrophic and the myometrium was normal.

\section{Discussion}

Extramammary Paget disease is an intraepithelial adenocarcinoma which usually is usually manifested in elderly women as an eczematous, crusted skin lesion in the vulval area. ${ }^{1}$ Histologically it is characterised by epidermal spread

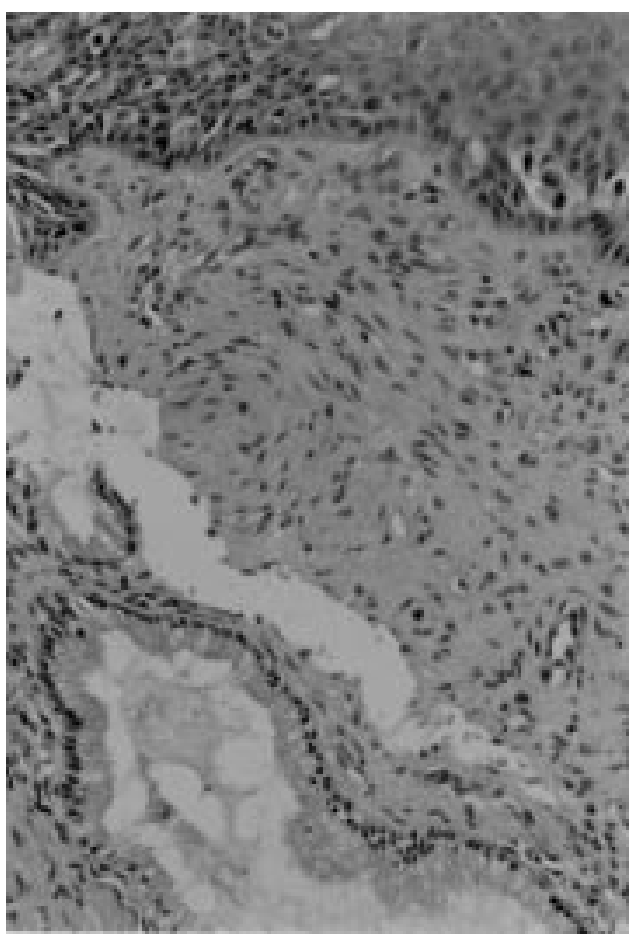

Figure 3 Extramammary Paget disease at the transformation zone of the cervix. (Haematoxylin and eosin, $\times 237$.) of large pale cells with atypical nuclei and cytoplasmic mucin.

Extramammary Paget disease is a rare neoplasm ( $1 \%$ of vulval tumours) which, in contrast to mammary Paget disease, is only relatively rarely associated with underlying in situ or invasive carcinoma. It occurs most commonly in the vulva but has been described in other sites where apocrine glands are present in high concentration including the axilla, the perianal area, and the eyelid, as well as in the male genitalia. In most cases where an associated neoplasm is present (either in situ or invasive) this is a cutaneous adnexal carcinoma, usually of apocrine or eccrine derivation. However, other primary malignancies more distant from the extramammary Paget disease lesion have also been described, including adenocarcinoma and transitional cell carcinoma of the bladder ${ }^{2}$ and adenocarcinoma of the cervix, anus, rectum, and prostate. In a significant proportion of cases of extramammary Paget disease an associated primary malignancy is not detected. In these cases, a separate histogenetic origin has been proposed, namely derivation from a pluripotent basal keratinocyte rather than epidermotropic spread from adjacent neoplastic disease. It is further postulated that, analogous to lentigo maligna, primary intraepidermal Paget disease (that is, without associated carcinoma) may eventually show dermal invasion after a significant period of intraepidermal growth and thus give rise to adenocarcinoma which may metastasise. $^{3}$

In our case it is most likely that the Paget disease arose secondary to the recurrent primary vulval adenocarcinoma (initially diagnosed eight years before this presentation). The symptoms of vulval irritation and discharge, which are those of Paget disease, were of relatively recent onset.

This case highlights important points about extramammary Paget disease of the vulva-its association with underlying carcinoma and its association with abnormalities in the squamous epithelium.

\section{ASSOCIATION WITH UNDERLYING CARCINOMA}

Studies have shown that the condition is associated with an underlying carcinoma in approximately $20 \%$ of cases. Hence a diagnosis of extramammary Paget disease should prompt a search for an underlying primary malignancy. Conversely, as demonstrated in this case, when a diagnosis of vulval adenocarcinoma is made it is advisable to draw the attention of the clinicians to the possibility of associated extramammary Paget disease, as its presence may alter subsequent patient management.

Extension of extramammary Paget disease of the vulva into the upper vagina and cervix has only rarely been described. ${ }^{145}$ Our case shows the need for full clinical assessment, including colposcopy and biopsy of suspicious mucosal lesions, when a diagnosis of vulval adenocarcinoma is made. It emphasises the point made by others that negative histological resection margins do not necessarily mean there has been complete excision. ${ }^{6}$ This case shows the 
importance of long term follow up of patients with Paget disease, even if initial surgical excision appears complete.

The treatment for Paget disease is surgical excision, although small recurrences or foci of residual disease, and more extensive lesions in those unsuitable for surgery, may be treated by laser vaporisation. The multifocal nature of the disease is well documented, and it has been shown that the histological extent of the disease extends far beyond the visible lesion in many cases. This has implications for the methods by which complete surgical excision is achieved, with some advocating the use of intraoperative frozen sections to achieve complete excision.

When a diagnosis of vulval adenocarcinoma is made, the possibility of coexisting extramammary Paget disease should always be considered and consultation with the clinicians is mandatory.

ABNORMALITIES IN THE SQUAMOUS EPITHELIUM

Extramammary Paget disease is often associated with abnormalities in the squamous epithelium. Striking epithelial hyperplasia, atypia, and keratinisation have been described and probably occur because of chronic irritation and trauma of the eczematous lesion, often exacerbated by a delay in diagnosis. ${ }^{8}$ Confusion between extramammary Paget disease and pagetoid Bowen disease can arise, but in this case the striking epidermal changes did not present a diagnostic problem since there had been previous histological confirmation of invasive adenocarcinoma. Without this information a superficial biopsy of the affected skin could easily be confused with atypical squamous disease of the vulva unless the diagnosis of extramammary Paget disease is kept in mind. Mucin stains are helpful in this regard.
There is a further possible area of confusion between Paget disease and malignant melanoma, ${ }^{9}$ as the latter is also characterised by intraepidermal invasion by large, atypical cells with a pale, granular cytoplasm. In difficult cases where this differential diagnosis is considered immunohistochemical staining is often helpful. The atypical cells in extramammary Paget disease usually show positive staining for epithelial membrane antigen (EMA), carcinoembryonic antigen (CEA), and Cam5.2 (less specific than CEA), while melanoma cells are negative for these markers but generally positive for HMB45 and S100.

Extramammary Paget disease should be considered as part of the differential diagnosis when atypical squamous disease of the vulva is encountered.

1 Jones RE, Austin C, Ackerman AB. Extramammary Paget's disease. A critical re-examination. Am $\mathcal{f}$ Dermatopathol disease. A critical

2 Hayashibara Y, Ikeda S. Extramammary Paget's disease with nternal malignancies. Gan To Kagaku Ryoho 1988;15: 1569-75

3 Evans AT, Neven P. Invasive adenocarcinoma arising in extramammary Paget's disease of the vulva. Histopathology 1991;18:355-60.

4 Costello TJ, Wang HH, Schnitt SJ, et al. Paget's disease with extensive involvement of the female genital tract initially detected by cervical cytosmear. Arch Pathol Lab Med 1988; 112:941-4.

5 Geisler JP, Gates RW, Shirrell W, et al. Extramammary Paget's disease with diffuse involvement of the lower female genito-urinary system. Int f Gynecol Cancer 1997;7:84-7.

6 Molinie V, Paniel BJ, Lessana-Leibowitch M, et al. Paget's Molinie V, Paniel BJ, Lessana-Leibowitch M, et al. Paget's
disease of the vulva. 36 cases. Ann Dermatol Venereol 1993; 120:522-7.

7 Curtin JP, Rubin SC, Jones WB, et al. Paget's disease of the vulva. Gynecol Oncol 1990;39:374-7.

8 Goldblum JR, Hart WR. Vulvar Paget's disease: a clinicopathologic and immunohistochemical study of 19 cases. Am $\mathcal{F}$ Surg Pathol 1997;21:1178-87.

9 Bacchi CE, Goldfogel GA, Greer BE, et al. Paget's disease and melanoma of the vulva. Use of a panel of monoclonal antibodies to identify cell type and to microscopically define adequacy of surgical margins. Gynecol Oncol 1992;46:216-21. 